\title{
AUTOCONOCIMIENTO, ADHERENCIA AL TRATAMIENTO Y CONTROL DE LA HIPERTENSIÓN ARTERIAL EN EL PERÚ: UNA REVISIÓN NARRATIVA
}

\author{
Percy Herrera-Añazco ${ }^{1,2, a}$, Josmel Pacheco-Mendoza2,b, Germán Valenzuela-Rodríguezª,d, Germán Málaga ${ }^{4,5, c}$
}

\begin{abstract}
RESUMEN
Se describe la producción científica peruana sobre hipertensión arterial (HTA) que se publicó en la literatura médica, mediante una revisión bibliográfica no sistemática hasta diciembre del 2016. Los resultados se dividieron en tres áreas temáticas: autoconocimiento, adherencia al tratamiento y control. Se encontraron 197 artículos, pero solo 15 se utilizaron para el análisis. Los resultados sugieren que el nivel de autoconocimiento de la HTA es deficiente, aunque ha mejorado en los últimos años. Esto es mejor en las poblaciones urbanas y en general, peor que en otras ciudades latinoamericanas. De igual forma, aunque el control de la HTA ha mejorado, es insuficiente y por debajo de otros países latinoamericanos. Finalmente, es posible que la adherencia al tratamiento sea peor en provincias.
\end{abstract}

Palabras clave: Hipertensión; Epidemiologia; Tratamiento farmacológico; Perú. (Fuente: DeCS, BIREME)

\section{SELF-KNOWLEDGE, ADHERENCE TO TREATMENT, AND CONTROL OF ARTERIAL HYPERTENSION IN PERU: A NARRATIVE REVIEW}

\begin{abstract}
This non-systematic bibliographic review examines the published Peruvian medical literature on arterial hypertension (HTN) as of December 2016. The results were divided into three thematic areas: self-knowledge, adherence to treatment, and control. We identified 197 articles, although only 15 were used for the analysis. Despite improvement in recent years, we found that the level of self-knowledge about HTN is poor. The level is better in urban areas, but is generally worse than in other Latin American cities. Moreover, despite improvement, HTN control is insufficient and worse than in other Latin American countries. Finally, adherence to treatment may be worse in the provinces.
\end{abstract}

Key words: Hypertension; Epidemiology; Drug therapy; Peru. (Source: MeSH, NLM)

\section{INTRODUCCIÓN}

La hipertensión arterial (HTA) es el principal factor de riesgo para el desarrollo de enfermedad cardiovascular (ECV) (1), la misma que en el 2012 representó el $31 \%$ de todas las muertes a nivel global ${ }^{(1,2)}$. Como consecuencia, la World Heart Federation ha emprendido una iniciativa para mejorar la tasa de control de la HTA a nivel mundial en $25 \%$ al $2025^{(3)}$.

Recientemente, una revisión sistemática calculó que para el 2010 existían 1,39 billones de personas con HTA a nivel mundial, lo que significó un aumento del $5,2 \%$ de la prevalencia de esta enfermedad en una década desde el 2000 al $2010^{(4)}$. Este aumento de la prevalencia no ha sido proporcional, reportándose una disparidad entre la prevalencia de la enfermedad entre países de altos ingresos y los de medianos a bajos ingresos. Pero quizá, lo más relevante, fue la disparidad entre el nivel de conocimiento, tratamiento y control de la HTA entre estos países, con un empeoramiento en el control de la HTA en los países de bajos ingresos en ese periodo (4).

Respecto a la prevalencia de HTA en Perú, Miranda JJ et al., comparó los factores de riesgo cardiovascular (FRCV) entre latinoamericanos y estadounidenses como

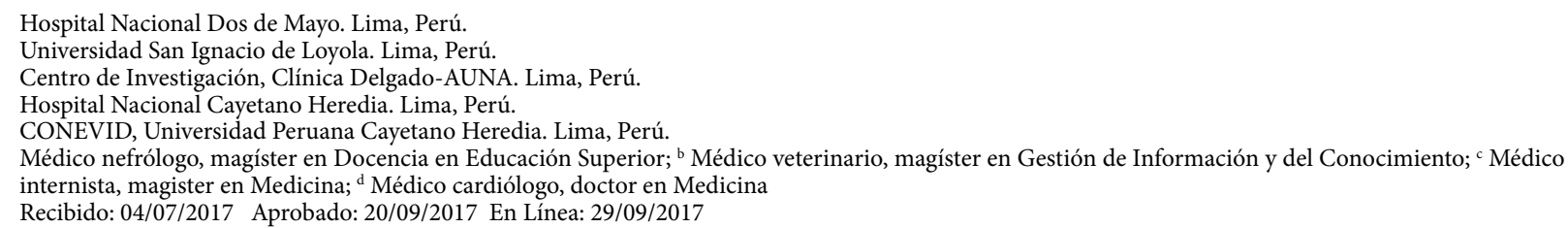

Citar como: Herrera-Añazco P, Pacheco-Mendoza J, Valenzuela-Rodríguez G, Málaga G. Autoconocimiento, adherencia al tratamiento y control de la hipertensión arterial en el Perú: una revisión narrativa. Rev Peru Med Exp Salud Publica. 2017;34(3):497-504.doi: 10.17843/rpmesp.2017.343.2622 
parte del The Latin American Consortium of Studies in Obesity (LASO). La prevalencia de HTA en general fue de $20 \%$ sin mostrar diferencias por sexo. El ratio de prevalencia ajustado para edad y sexo de HTA fue de 0,65 . En ese estudio, el mayor ratio de prevalencia fue para Venezuela con 1,49 , y el menor para Argentina con $0,61^{(5)}$. Asimismo, en población adulta mayor, en el 2015 , Enrique Ruiz Mori et al. presentó los resultados de una evaluación FRCV entre pacientes limeños de más de 80 años. La prevalencia de HTA fue de $44 \%$, siendo más común entre las mujeres $(62,2 \%)$. La HTA fue el FRCV más frecuente en la población estudiada ${ }^{(6)}$.

Entre los países de bajos ingresos, muchos son los factores de riesgo asociados a una mayor prevalencia de HTA, tales como: edad mayor de 65 años, personas sin educación formal, pacientes obesos con sobrepeso, pacientes en poblaciones urbanas, sexo femenino y consumo de alcohol ${ }^{(7,8)}$. Asimismo, algunos autores han sugerido que aspectos relacionados con la deficiente salud perinatal en países del tercer mundo y problemas relacionados con los sistemas de salud son factores asociados a la alta prevalencia, así como al pobre control, no adherencia al tratamiento y desconocimiento de la enfermedad ${ }^{(9,10)}$. El Perú es un país con una economía en transición, con alta prevalencia de los factores de riesgo conocidos para el desarrollo y pobre control de la HTA, lo que, sumado al bajo nivel educativo con su consecuente pobre nivel de conocimiento de la enfermedad y de las implicancias del tratamiento, constituyen un caldo de cultivo para el desarrollo y evolución natural de la misma ${ }^{(11)}$.

La importancia de la HTA, asociada a la gran carga de la misma, su implicancia en la afección de la calidad de vida y el ser causante de discapacidad, requiere que la comunidad médica esté preparada para su manejo. Por tal motivo, la investigación local para entender factores asociados a mala evolución de la enfermedad es prioritaria. En los últimos años se han publicado una serie de artículos sobre HTA en población peruana, por lo que es necesario sistematizar esta información para el conocimiento de la comunidad médica.

En ese sentido, el objetivo del presente artículo es desarrollar una revisión narrativa de la producción científica publicada en la literatura médica sobre HTA relacionada a aspectos de autoconocimiento, adherencia al tratamiento y control de la HTA en población peruana con dirección y filiación Perú hasta diciembre de 2016. De tal forma que nuestros hallazgos pueden servir a los tomadores de decisión a orientar las intervenciones en salud pública hacia los puntos que la evidencia nacional muestre mayores deficiencias.

\section{METODOLOGÍA}

Se desarrolló una búsqueda en las siguientes bases de datos: Web of Knowledge: Web of Science Core
Collection (WoS), SciELO Citation Index y MEDLINEWoS; LILACS; Elsevier - SCOPUS y Google Scholar. Los resultados se presentan en áreas temáticas preestablecidas: autoconocimiento, tratamiento $\mathrm{y}$ control de la HTA.

Se utilizó la siguiente fórmula para las bases de Clarivate Analytics (ex Thomson Reuters), período $=1950-2016$ :

WoS: TS= ("Arterial Hypertension") AND (CU=PERU ORAD=PERU) Medline: (TS= ("Arterial Hypertension") OR MH=(Hypertension)) AND AD=PERU

Scielo Citation Index: TS= ("Arterial Hypertension") AND CU=PERU

Y para Scopus (Elsevier), periodo $=1950-2016$ :

(TITLE-ABS-KEY (“Arterial Hipertensión”) AND AFFILCOUNTRY (PERU) AND PUBYEAR < 2017)

La búsqueda corrió a cargo de 02 investigadores, y de la búsqueda completa se obtuvieron de WoS, Medline, SciELO Citation Index, SCOPUS y LILACS: 22, 36, 35, 30, 88 documentos respectivamente. La revisión de los textos estuvo a cargo de todos los investigadores. Después de la selección, los artículos se redujeron a 15, ya que estaban referidos a las áreas temáticas preestablecidas y sirvieron para el desarrollo de la presente revisión.

\section{RESULTADOS}

\section{AUTOCONOCIMIENTO DE LA HIPERTENSIÓN ARTERIAL EN EL PERÚ}

El autoconocimiento de la HTA es una tarea pendiente en nuestra población. Los resultados de los estudios que han evaluado el tema en nuestra población, reportan en la mayoría de los casos que éste es menor al $50 \%$, valores menores que los reportados en otras ciudades de Latinoamérica. Sin embargo, no siempre son estudios comparables debido a lo heterogéneo de las poblaciones estudiadas y la metodología empleada. Los principales resultados los presentamos a continuación en forma cronológica.

Seclén et al. (1999), en un estudio de 230660 personas mayores de 18 años divididas en cuatro poblaciones urbanas; dos de la costa (Piura y Lima), de la selva (San Martín y Ancash) y dos comunidades rurales de la selva (San Martín), no proporcionó información precisa sobre autoconocimiento de la HTA, ni la forma como se evaluó. Sin embargo, reportaron que el nivel de autoconocimiento fue bajo y en el gráfico mostrado en su artículo, los rangos varían entre 1 a $12 \%$, aproximadamente ${ }^{(12)}$.

En el estudio PREVENCION (2009), realizado en 1878 personas de la ciudad de Arequipa, la proporción general 
de sujetos hipertensos que conocían su diagnóstico fue de 47,9\% (IC 95\% = 42,5-53,4\%). Asimismo, el porcentaje de autoconocimiento del diagnóstico fue de $33,4 \%$ (IC $95 \%=23,6-44,9 \%$ ) para sujetos hipertensos menores de 50 años y de $54,8 \%$ (IC 95\%=49,1-60,3\%) para aquellos mayores de 50 años. No hubo diferencia significativa en relación al sexo ${ }^{(13)}$.

En el 2009, Hernández-Hernández et al., como parte del CARMELA study, evaluaron el nivel de autoconocimiento de la HTA en siete ciudades de Latinoamérica que incluía cerca de 1600 participantes en Lima. En este estudio, el $47 \%$ de los limeños evaluados no sabían que tenían HTA y era la ciudad con el menor porcentaje de autoconocimiento de la enfermedad respecto al resto de ciudades que incluían: Barquisimeto, Bogotá, Buenos Aires, Ciudad de México, Quito, y Santiago de Chile ${ }^{(14)}$.

En el 2011, en el estudio TORNASOL II (14), los autores encontraron que el nivel de autoconocimiento de la HTA fue de $48,2 \%$, siendo mayor que en el estudio TORNASOL I (2006) donde el nivel de autoconocimiento fue de $44,9 \%{ }^{(13)}$. Así mismo, hubo una mejora en la frecuencia de autoconocimiento según región del Perú. En la costa de 49 a $52,4 \%$, en la sierra de 40,1 a $42 \%$ y en la selva de 43 a $59,5 \%{ }^{(15,16)}$.

En el estudio multinacional de Prince MJ et al. (2011), en pacientes mayores de 65 años en poblaciones rurales y urbanas de Latinoamérica, China e India, que incluye población urbana (Lima) y rural (Cañete) de Perú, los autores señalan que, respecto al resto de países evaluados, los pacientes de Perú y Puerto Rico destacaban en el área de autoconocimiento de la HTA (Perú rural 94\% y Perú urbano 92,4\%). Resultados discrepantes con respecto al resto de estudios que sobre el tema se han hecho en población peruana. En dicho estudio se señala, además, que los niveles de autoconocimiento son deficientes en la India (India rural $27,8 \%$ e India urbana $38,9 \%$ ) o China (China rural $44,6 \%$ y China urbana $77,6 \%$ ), entre otros países ${ }^{(17)}$.

En el MIGRANT STUDY (2013), que evalúa a pacientes rurales de Ayacucho, migrantes ayacuchanos en Lima y urbanos de Lima, se reporta que el $48,3 \%$ de la población general conocía de su enfermedad. Según el tipo de población, el autoconocimiento fue de $36,4 \%$ en la población rural, $55,7 \%$ entre los migrantes y $42,4 \%$ en la población urbana ${ }^{(18)}$.

En el 2014, Aguado-Fabián et al., reportaron el nivel de autoconocimiento y autocuidados entre 86 pacientes que acudieron a consultorios de cardiología del Hospital Nacional Cayetano Heredia. El 40,0\% de los pacientes tenían un nivel de conocimiento y autocuidado alto $\mathrm{y}$ el $38,1 \%$ presentaron un nivel de conocimiento $y$ autocuidado bajo ${ }^{(19)}$.
Finalmente, en el 2016 una muestra de participantes del CRONICAS Cohort Study ${ }^{(20)}$, estudio que incluyó 3601 personas de Tumbes, Puno y Lima. Se encontró que de 717 pacientes con HTA, el $28 \%$ sabía de su condición de hipertenso. El desconocimiento de su estatus de hipertenso era mayor en Tumbes (41\%) que en Lima (31\%) y mucho menor tanto en el área rural de Puno $(21 \%)$ y en área rural de la misma ciudad (7\%). Asimismo, era menor en pacientes con bajo estatus socioeconómico (39\%) que entre los de ingresos medios y altos (29 y $31 \%$ respectivamente) ${ }^{(20)}$.

\section{TRATAMIENTO Y CONTROL DE LA HIPERTENSIÓN}

Probablemente, los trabajos más completos que han incluido datos clínicos y epidemiológicos de la hipertensión en el Perú son los estudios TORNASOL I (2006) y TORNASOL II (marzo 2010-Enero $2011^{(15,16)}$. En dichos estudios se muestra una mejora tanto en el tratamiento y control de la HTA en las poblaciones evaluadas.

En el estudio TORNASOL I, se describió, entre otros aspectos, el tratamiento y control de los pacientes peruanos, clasificándolos por regiones: costa, sierra y selva. Del total de pacientes, el 32,7\% recibía tratamiento antihipertensivo: en la costa fue el $37,9 \%$, en la sierra el $27,2 \%$ y en la selva el $27,9 \%{ }^{(15)}$. Sin embargo, del total de pacientes hipertensos, solo el 14,7\% tuvo HTA controlada: en la costa fue el $16,2 \%$, en la sierra el $12,9 \%$ y en la selva el $14,7 \%{ }^{(15)}$.

En el estudio TORNASOL I se consignó que la mayoría de hipertensos recibió inhibidores de la enzima convertidora de angiotensina (IECA) (82,2\%), seguidos por los calcioantagonistas dihidropiridínicos (12\%) y luego por otras clases de medicamentos hipotensores. Asimismo, este esquema terapéutico se repitió en las tres regiones del País, según los datos de Agusti (2006) ${ }^{(15)}$.

Algunos años después, en el estudio TORNASOL II (2011), realizado con la misma metodología, se encontró que el porcentaje de pacientes tratados a nivel nacional fue de $39,3 \%$ : en la costa de $44,1 \%$, en la sierra de $32,6 \%$ y en la selva de $39,8 \%$. Asimismo, del total de pacientes hipertensos, solo el $20,6 \%$ tuvo HTA controlada: en la costa fue de $22,8 \%$, en la sierra de $17,3 \%$ y en la selva de $21,4 \%{ }^{(16)}$.

Otros estudios que han evaluado tanto el tratamiento y control de la HTA en el Perú, muestran resultados diversos, aunque al parecer peores que los encontrados en otras ciudades de Latinoamérica. Estos resultados son heterogéneos debido al tipo de población estudiada y que a continuación mostramos en forma cronológica. Rivas-Chávez et al. (2008) realizaron un estudio 
reportando los patrones de tratamiento en 865 pacientes del Hospital PNP Augusto B. Leguía, cuya edad promedio fue de $63,5 \pm 12,2$ años y el $54,5 \%$ era de sexo masculino. Los fármacos más usados fueron el enalapril $(56,61 \%)$, seguido por los calcio antagonistas dihidripiridínicos (amlodipino, 16,87\% y nifedipino, $5,05 \%$ ), los betabloqueadores (atenolol $9,79 \%$ ) y los diuréticos tiazídicos $(9,60 \%){ }^{(21)}$.

El $50 \%$ de los pacientes recibía un solo fármaco, el $43 \%$ dos fármacos y el $7 \%$ tres o más fármacos, siendo la combinación más frecuente el enalapril y la hidroclorotiazida, encontrándose que a mayor estadio de HTA se incrementaba la cantidad de fármacos prescritos ${ }^{(21)}$. El grado de control fue de $89,1 \%$ (en los no-diabéticos de $91,8 \%$ y en los diabéticos de $63,4 \%$, $p<0,001)$, encontrándose que el atenolol y el enalapril serían, probablemente, los más costo-efectivos al valorarse la relación entre el costo de los medicamentos y su prescripción ${ }^{(21)}$.

En el estudio PREVENCION (2009), la proporción de pacientes que recibían tratamiento farmacológico fue $39,5 \%$, sin diferencias por sexo, pero que se incrementó con la edad: $24,9 \%$ en menores de 50 años, hasta $46,4 \%$ en mayores de 50 años.

De los pacientes que recibían terapia farmacológica, el $35,7 \%$ tuvieron HTA controlada, que representó el $14 \%$ de todos los hipertensos. La proporción de pacientes hipertensos con HTA controlada no difirió por sexo, pero la tasa de control disminuyó significativamente con una mayor edad: $51,4 \%$ en menores de 50 años hasta $31,8 \%$ en mayores de 50 años ${ }^{(13)}$.

En el CARMELA study (2009), de los cerca de 1600 de limeños evaluados, el $61 \%$ usaba medidas no farmacológicas, $28,8 \%$ usaba medidas farmacológicas, pero solo el $12 \%$ tenía la HTA controlada. Tanto en uso de medidas farmacológicas y control de la HTA, Lima era la ciudad con peor control respecto al resto de ciudades evaluadas ${ }^{(22)}$.

El estudio multinacional de Prince et al. (2011), al igual que el aspecto de autoconocimiento, se encontró resultados discrepantes respecto a otros estudios sobre el tema. En este estudio, se encontró una tasa de tratamiento de $88,5 \%$ para los pacientes de Lima y de $72,9 \%$ para el Perú rural, entre la subpoblación de pacientes que tenían conocimiento de su enfermedad. Por otro lado, el porcentaje de control en el Perú urbano fue de $76,9 \%$ y en el Perú rural de $89,5 \%{ }^{(17)}$. Es probable, que sesgos metodológicos expliquen estos resultados aparentemente alentadores en este rubro tanto como en el de autoconocimiento.
Lerner et al. (2013) en su reporte sobre HTA del MIGRANT STUDY, encuentran que en la población urbana el conocimiento de la hipertensión fue de $48,3 \%$. De ellos, el $40 \%$ de pacientes eran tratados y de ellos estaban en control el 30,4\%. En la población rural, el conocimiento de la hipertensión fue de 36,4\%. De ellos, el 16,7\% estaban tratados y ninguno estaba controlado. Finalmente, en la población de migrantes rural-urbano, el 55,7\% conocía su enfermedad, pero el $30,5 \%$ recibía tratamiento y de ellos, el 33,3\% estaba controlado ${ }^{(22)}$. Con estos datos se demostró que la regla de las mitades no aplica para algunas poblaciones de Perú, sobre todo para los migrantes ruralurbano y los habitantes de zonas rurales ${ }^{(18)}$.

Por último, en el reporte del CRONICAS Cohort Study en el $2016{ }^{(20)}$, el $30 \%$ de los participantes sabían de su condición de hipertensos, pero no estaban tratados. A los 15 meses de seguimiento el 56,9\% recibían tratamiento y solo el $25,4 \%$ estaban controlados. Los factores predictores positivos para recibir tratamiento fueron la edad y la historia familiar de enfermedades crónicas ${ }^{(20)}$ (Tabla 1).

\section{TRATAMIENTO Y CONTROL DE LA HIPERTENSIÓN EN POBLACIONES ESPECIALES}

Los estudios encontrados en este rubro son los hechos en pacientes adultos mayores y en gestantes.

Ruiz-Mori et al. (2015) evaluaron a 969 individuos mayores de 80 años de edad en la ciudad de Lima y la provincia constitucional del Callao. En esta población, el $87 \%$ se encontraba en tratamiento farmacológico, $65 \%$ de los cuales estaban controlados. En el grupo de pacientes hipertensos que no utilizaban tratamiento farmacológico, un $56 \%$ tenía un control de la HTA ${ }^{(6)}$.

Adicionalmente, Bravo J et al. (2013) reportaron un estudio realizado en las gestantes con HTA crónica tratadas en el Hospital Nacional Cayetano Heredia cuyos datos se recolectaron entre el 2006 y el 2012, encontrándose que la mayoría recibía tratamiento antihipertensivo con base en un medicamento (39\%), dos medicamentos $(36,6 \%)$ y tres o más medicamentos (14,6\%). El tiempo de tratamiento fue durante todo el embarazo en el $48,8 \%$ y en el periparto en el $51,2 \%$. Los antihipertensivos usando con más frecuencia fueron alfa metildopa, amlodipino y nifedipino ${ }^{(23)}$.

La HTA crónica se presentó aislada en el 31,7\% de casos y asociada a preeclampsia leve en el $14,6 \%$ y a preeclampsia severa en el $43,9 \%$. Los casos de hipertensión más preeclampsia severa y síndrome Hellp se presentaron en el $9,8 \%{ }^{(23)}$.

\section{ADHERENCIAAL TRATAMIENTOANTIHIPERTENSIVO}

Respecto a la adherencia al tratamiento, al igual que en los estudios mostrados en las áreas temáticas previas, hay 
heterogeneidad en la forma en que fue evaluada. A pesar de esto, es claro que este aspecto es un área a trabajar y, posiblemente, en provincia este problema sea mayor.

Carhuallanqui et al. (2010) entrevistaron a una población de pacientes hipertensos atendidos en el Hospital Cayetano Heredia, utilizando la prueba de MoriskyGreen para determinar su adherencia al tratamiento farmacológico. Ciento tres pacientes fueron estudiados, siendo predominante en mayores a 60 años $(80,6 \%)$ y en sexo femenino $(69,9 \%)$. El control de la presión arterial se encontró en el $62,1 \%$ de pacientes y la adherencia al tratamiento farmacológico fue de $37,9 \%{ }^{(24)}$.

Otros datos relacionados con la adherencia al tratamiento fueron los de Fernández-Arias (2014), quien encuestó a 115 pacientes atendidos en el Hospital Cayetano. La edad promedio fue de $62,7 \%$ y $77(67 \%)$ eran de sexo femenino. Ciento dos $(88,7 \%)$ venían siendo tratados por más de 6 meses, con uno a dos medicamentos $(47,8 \%)$ y con tres a más $(52,2 \%)$. Se encontró una baja adherencia a la medicación según los resultados de la escala Morisky en el $57,4 \%$ de los pacientes, predominantemente en jóvenes y en aquellos a quienes se les habían prescrito más de dos medicamentos ${ }^{(25)}$.

Esta baja adherencia se asociaría, sin duda, a un bajo nivel de conocimiento y autocuidado de su enfermedad.
Aguado-Fabián et al. (2014) realizaron entrevistas predeterminadas a 86 pacientes hipertensos del Hospital Nacional Dos de Mayo, encontrando que el nivel de conocimiento sobre hipertensión arterial en los pacientes fue medio $(46,5 \%)$, bajo $(24,4 \%)$ y alto $(29,1 \%)$. Adicionalmente, al estudiar el autocuidado de los pacientes se encontró un nivel medio en el $58,2 \%$, bajo en el $15,2 \%$ y alto en el $26,7 \%{ }^{(19)}$.

En Chiclayo, en un estudio que evaluó la "Cartera de Servicios de Salud de Complejidad Creciente del Policlínico Chiclayo Oeste" en el 2015 , solo el $11 \%$ de los pacientes evaluados eran adherentes a su tratamiento antihipertensivo, la misma que se evaluó usando el cuestionario "Martin Bayarre Grau". Asimismo, solo el $18,7 \%$ cumplian su tratamiento higiénico dietético, y casi la mitad tenía un grado de instrucción superior (26).

\section{DISCUSIÓN}

Los principales hallazgos de nuestro estudio muestran que el nivel de autoconocimiento de la HTA es aún deficiente. En cuanto a su distribución, el autoconocimiento es mejor en las áreas urbanas que en las rurales. De igual forma, aunque el tratamiento, y control de la HTA ha mejorado, aun es insuficiente $y$, en todos los aspectos mencionados, estamos en desventaja con relación a otras ciudades latinoamericanas.

Tabla 1. Estudios que evalúan el tratamiento, control o variables relacionadas de la hipertensión arterial en el Perú

\begin{tabular}{|c|c|c|c|c|c|c|}
\hline Año & Autores & $\mathbf{N}^{\circ}$ & Tipo de estudio & Tratamiento & Control & Localidad \\
\hline 2015 & Ruiz-Mori et al. & 969 & $\begin{array}{l}\text { Descriptivo- } \\
\text { transversal }\end{array}$ & $87 \%$ & $65 \%$ & Lima Metropolitana \\
\hline 2015 & Bilo et al. & 100 & Ensayo clínico & DNP & DNP & $\begin{array}{l}\text { Lima Metropolitana } \\
\text { Ilevados a gran } \\
\text { altura ( } 3260 \mathrm{~m} \text { de } \\
\text { altitud) }\end{array}$ \\
\hline 2014 & $\begin{array}{l}\text { Fernández-Arias } \\
\text { et al. }\end{array}$ & 115 & $\begin{array}{l}\text { Descriptivo- } \\
\text { transversal }\end{array}$ & Adherencia $(57,4 \%)$ & DNP & Lima Metropolitana \\
\hline 2014 & Aguado-Fabián & 86 & $\begin{array}{l}\text { Descriptivo- } \\
\text { transversal }\end{array}$ & DNP & DNP & Lima Metropolitana \\
\hline 2013 & $\begin{array}{l}\text { Segura-Vega, } \\
\text { Agusti, Ruiz-Mori } \\
\text { et al. }\end{array}$ & $\begin{array}{r}14 \\
508\end{array}$ & $\begin{array}{l}\text { Descriptivo- } \\
\text { transversal }\end{array}$ & $\begin{array}{c}32,7 \% \text { en Tornasol I y } 39,3 \% \text { en } \\
\text { Tornasol II }\end{array}$ & $\begin{array}{l}14,7 \% \text { en Tornasol I y } \\
20,6 \% \text { en Tornasol II }\end{array}$ & $\begin{array}{c}26 \text { ciudades } \\
\text { de algunos } \\
\text { departamentos del } \\
\text { Perú }\end{array}$ \\
\hline 2013 & Bravo et al. & 41 & $\begin{array}{l}\text { Descriptivo- } \\
\text { transversal }\end{array}$ & DNP & DNP & Lima Metropolitana \\
\hline 2013 & Lerner et al. & 987 & $\begin{array}{l}\text { Descriptivo- } \\
\text { transversal }\end{array}$ & $\begin{array}{c}\text { Urbano }(50 \%), \text { rural }(16,7 \%), \\
\text { migrantes }(30,5 \%)\end{array}$ & $\begin{array}{c}\text { Urbano }(28,6 \%) \text {, rural } \\
(0 \%), \text { migrantes }(33 \\
, 3 \%)\end{array}$ & Ayacucho y Lima \\
\hline 2011 & Prince et al. & 134 & $\begin{array}{l}\text { Descriptivo- } \\
\text { transversal }\end{array}$ & $\begin{array}{l}88,5 \% \text { en Perú urbano y } 72,9 \% \text { en } \\
\text { Perú rural }\end{array}$ & $\begin{array}{l}76,9 \% \text { en Perú urbano } \\
\text { y } 89,5 \% \text { en Perú rural }\end{array}$ & $\begin{array}{c}\text { Perú urbano y Perú } \\
\text { rural }\end{array}$ \\
\hline 2010 & Carhuallanqui et al. & 103 & $\begin{array}{l}\text { Descriptivo- } \\
\text { transversal }\end{array}$ & DNP & $\begin{array}{l}37,9 \% \text { con adherencia } \\
\text { al tratamiento de } 62,1 \%\end{array}$ & Lima Metropolitana \\
\hline 2008 & $\begin{array}{l}\text { Rivas-Chávez } \\
\text { et al. }\end{array}$ & 865 & $\begin{array}{l}\text { Descriptivo- } \\
\text { transversal }\end{array}$ & DNP & DNP & Lima Metropolitana \\
\hline 2007 & $\begin{array}{l}\text { Medina-Lezama, } \\
\text { Zea-Díaz et al. }\end{array}$ & 1878 & $\begin{array}{l}\text { Descriptivo- } \\
\text { transversal }\end{array}$ & $39,5 \%$ & $14 \%$ & Arequipa \\
\hline
\end{tabular}

DNP: Dato no disponible 


\section{POBRE AUTOCONOCIMIENTO DE LA HTA. UNA OPORTUNIDAD PARA MEJORAR}

Aunque en nuestra población el autoconocimiento es deficiente, los datos muestren que ha habido una mejora a través de los años, según los resultados de los estudios Tornasol I y Tornasol II (15,16). Sin embargo, respecto a otras ciudades latinoamericanas, aún hay trabajo por hacer ${ }^{(14)}$.

A nivel mundial, el autoconocimiento de la HTA en los países de medianos y bajos ingresos no solo está muy por debajo de los países de altos ingresos, sino que la gradiente de mejora de estos rubros a través de los años ha sido mayor entre los países de altos ingresos respecto a los de medianos y bajos ingresos. Mientras que en los países de altos ingresos este rubro mejoró de 58,2 a $67,0 \%$, en los países de medianos y bajos ingresos, solo aumentó de 32,3 a $37,9 \%{ }^{(4)}$. Sin embargo, entre países de bajos ingresos, existe variación. En un estudio hecho en 35125 pacientes de más de 50 años en China, Ghana, India, México, La Federación Rusa y Sud África, el autoconocimiento fue en promedio del $48,3 \%$ con rangos que variaban entre $23,3 \%$ en Ghana a $72,1 \%$ en la Federación Rusa ${ }^{(27)}$. No obstante, el autoconocimiento de la HTA puede ser menor si es que la evaluación se realiza en población pobre de ámbitos rurales, como se encontró en el MIGRANT STUDY (22) o en un reciente reporte colombiano, donde el vivir en áreas rurales y tener bajo nivel educativo estuvo asociado a pobre autoconocimiento de la HTA ${ }^{(28)}$.

Estos hallazgos no son exclusivos de la HTA, ya que lo mismo se ha encontrado en otros FRCV. Por ejemplo, en un estudio que evaluó el tema en 12 países de medianos y bajos ingresos se encontró que el autoconocimiento de la diabetes fue del $69 \%$ en la población general y $80 \%$ en la población de alto riesgo para esta enfermedad. Asimismo, el autoconocimiento de la enfermedad renal crónica fue de $6 \%$ en la población general y $10 \%$ en la población de alto riesgo para esta enfermedad ${ }^{(29)}$.

Se refuerza, por lo tanto, la idea de que nuestras autoridades tienen un amplio camino por recorrer para cerrar esta brecha.

\section{TRATAMIENTO Y CONTROL DE LA HIPERTENSIÓN ARTERIAL: MUCHO POR HACER}

Los estudios que han evaluado el nivel de tratamiento y control de la HTA en el Perú, reportan que, en estos aspectos, menos del $50 \%$ de la población tienen niveles óptimos de tratamiento y control de la presión arterial, situación que los hace vulnerables al desarrollo de complicaciones serias, sino fatales, como consecuencia de infartos de miocardio, accidentes cerebrovasculares, entre otras complicaciones ${ }^{(6,13,15,16-28,19,21,22-25) \text {. }}$
A nivel internacional, se ha apreciado una mejora sostenida tanto a nivel de tratamiento $(44,5 \%$ a $55,6 \%$ ) y control de la HTA $(17,9 \%$ a $28,4 \%)$ en países de altos ingresos entre el 2000 al 2010. Mientras que, en países de medianos y bajos ingresos, esta mejora ha sido discreta en cuanto al tratamiento $(24,9$ a $29 \%$ ) y ha disminuido en cuanto el control de la HTA $(8,4 \text { a } 7,7 \%)^{(4)}$. En nuestro país, los estudios TORNASOL I y II mostraron que hubo una mejora de $6,6 \%$ respecto al tratamiento y de $5,9 \%$ respecto al control de la HTA ${ }^{(15,16)}$, con datos absolutos mejores que los vistos en una reciente revisión sistemática ${ }^{(4)}$. De igual forma, en el recientemente publicado "Perú: enfermedades no transmisibles y transmisibles, 2016" (INEI 2016), aunque se reporta que en el 2016 hubo una disminución de 0,9\% del número de pacientes hipertensos diagnosticados por un médico respecto al 2016, hubo una mejora del 3,9\% de pacientes que compraron antihipertensivos en el mismo periodo ${ }^{\left({ }^{30}\right)}$. No obstante, al igual que en el rubro de autoconocimiento, nuestros niveles están por debajo de lo encontrado en otras ciudades latinoamericanas ${ }^{(22) .}$

Al igual que con el autoconocimiento, el control de la HTA, si bien variable, es uniformemente deficientes en países de bajos ingresos. En la evaluación de Lloyd-Sherlock $\mathrm{P}$ et al., el control de la HTA promedio fue de $10,2 \%$, pero con rangos de $4,1 \%$ en Ghana a $14,1 \%$ en India ${ }^{(8)}$. Es necesario tener en cuenta que tanto el tratamiento como control de la HTA puede variar dependiendo de la región en la que es evaluada, como se mostró en nuestro país en el MIGRANT STUDY o en el TORNASOL I y || $(15,16,18)$. Incluso, en Estados Unidos existen variaciones étnicas registradas, como en el estudio de Sorlie PD et al., donde se mostró un menor tratamiento y control de la HTA entre hispanos respecto a los blancos no hispanos ${ }^{(31)}$. Es posible que aspectos relacionados a inequidad en los sistemas de salud pueden estar relacionadas ${ }^{(10)}$.

\section{FORTALEZAS Y LIMITACIONES}

Esta es una revisión narrativa con algunas limitaciones. En primer lugar, solo se han incluido datos de estudios publicados en revistas científicas, pudiendo existir datos de tesis que podrían complementar algunos aspectos relacionados de la HTA en el país. Sin embargo, el usar solo artículos de revistas, nos garantiza una revisión por pares independiente que podría mejorar la calidad de los datos analizados.

En segundo lugar, los estudios incluidos tienen serias limitaciones metodológicas que incluyen los siguientes aspectos: algunos estudios son de un solo centro, tienen tamaños de muestra reducidas y en algunas circunstancias esta fue tomada por conveniencia, no siendo generalizables y que podrían explicar algunos hallazgos muy disimiles entre los estudios ${ }^{(17)}$.

A pesar de ello, es la primera revisión de este tipo sobre la HTA en el Perú, que evalúa aspectos de autoconocimiento, tratamiento y control que incluye estudios actuales y nos 
permite tener una visión integral sobre puntos importantes a evaluar en futuras investigaciones con mejor diseño metodológico.

\section{CONCLUSIONES}

El tratamiento y el control de la HTA han mejorado en los últimos años, aunque estos aún son deficientes y muy por debajo del promedio de los países de altos ingresos y otros países latinoamericanos. La adherencia al tratamiento en HTA, según la información disponible, es también deficiente.

El Ministerio de Salud, y las instituciones científicas del país deberían diseñar investigaciones, intervenciones y estrategias con adecuada calidad metodológica, que permitan mejorar el conocimiento de los diferentes aspectos de la HTA en el Perú, que son perfectibles y han sido identificados en nuestra revisión.

Contribuciones de los autores: PHA y JPM han participado en la concepción del artículo. JPM participó en la recolección de datos. GM, PHA y GV realizaron el análisis de datos. Todos los autores participaron en la redacción y la aprobación de la versión final

Conflictos de interés: no existen conflictos de interés en la publicación de este artículo.

Fuentes de financiamiento: autofinanciado

\section{REFERENCIAS BIBLIOGRÁFICAS}

1. World Health Organization. WHO | Cardiovascular diseases (CVDs) 2017. http:// www.who.int/mediacentre/factsheets/ fs317/en/ (accessed mayo 15, 2017).

2. World Heart Federation. 2015. Cardiovascular disease risk factors. [online] Available at: http://www.world-heartfederation.org/cardiovascular- health/ cardiovascular-disease-risk-factors/ (Accessed 15/11/2016).

3. Adler AJ, Prabhakaran D, Bovet P, Kazi DS, Mancia G, Mungal-Singh V, et al. Reducing cardiovascular mortality through prevention and management of raised blood pressure: a World Heart Federation Road Map. Glob Heart 2015; 10: 111-22.

4. Mills KT, Bundy JD, Kelly TN, Reed JE, Kearney PM. Global Disparities of Hypertension Prevalence and Control: A Systematic Analysis of Population-Based Studies From 90 Countries. Circulation. 2016 Aug 9;134(6):441-50.

5. Miranda JJ, Herrera VM, Chirinos JA, Gómez LF, Perel P, Pichardo R, et al. Major cardiovascular risk factors in Latin America: a comparison with the United States. The Latin American Consortium of Studies in Obesity (LASO). PLoS One. 2013;8(1):e54056.

6. Ruiz Mori E, Ruiz M H, Guevara Gonzales L, Ortecho Arias H, Salazar Rojas R, et al. Factores de riesgo cardiovascular en mayores de 80 años. Horiz Med.2015; 15(3): 26-33.

7. Sarki AM, Nduka CU, Stranges S, Kandala NB, Uthman OA. Prevalence of Hypertension in Low- and Middle-
Income Countries: A Systematic Review and Meta-Analysis. Medicine (Baltimore). 2015 Dec;94(50):e1959.

8. Lloyd-Sherlock P, Beard J, Minicuci N, Ebrahim S, Chatterji S. Hypertension among older adults in low- and middleincome countries: prevalence, awareness and control. Int J Epidemiol. 2014 Feb;43(1):116-28.

9. Mittal BV, Singh AK. Hypertension in the Developing World: Challenges and Opportunities. Am J Kidney Dis. 2010 Mar;55(3):590-8.

10. Maimaris W, Paty J, Perel P, LegidoQuigley $\mathrm{H}$, Balabanova $\mathrm{D}$. The Influence of Health Systems on Hypertension Awareness, Treatment, and Control: A Systematic Literature Review. PLoS Med. 2013;10(7):e1001490.

11. Ministerio de Salud, Dirección General de Epidemiología. 2013. Análisis de situación de salud del Perú. [online] Available at: http://www.dge.gob.pe/portal/ docs/intsan/asis2012.pdf. (Accessed $15 / 11 / 2016$ ).

12. Seclén $S$, Leey J, Villena A, Herrera B, Menacho J, et al. Prevalencia de obesidad, diabetes mellitus, hipertensión arterial e hipercolesterolemia como factores de riesgo coronario y cerebrovascular en población adulta de la costa, sierra y selva del Perú. Acta Médica Peruana. 1999; 17 (1): 8-12.

13. Medina-Lezama J, Chirinos JA, Zea Díaz $\mathrm{H}$, Morey O, Bolanos JF, Munoz-Atahualpa E, et al. Design of PREVENCION: a populationbased study of cardiovascular disease in Peru. Int. J. Cardiol. 2005;105(2):198-202.
14. Hernández-Hernández R, Silva $\mathrm{H}$ Velasco M, Pellegrini F, Macchia A, et al. Hypertension in seven Latin American cities: the Cardiovascular Risk Factor Multiple Evaluation in Latin America (CARMELA) study. J Hypertens. 2010 Jan;28(1):24-34.

15. Segura Vega L, Agusti C R, Parodi J. Factores de Riesgo de las Enfermedades Cardiovasculares en el Perú. Estudio TORNASOL. Revista Peruana de Cardiología. 2006;32(2):82-128.

16. Segura Vega L, Agusti C R, Parodi J. Factores de Riesgo de las Enfermedades Cardiovasculares en el Perú. Estudio TORNASOL. Revista Peruana de Cardiología. 2011;37(1):19-27.

17. Prince MJ, Ebrahim S, Acosta D, Ferri CP, Guerra M, et al. Hypertension prevalence, awareness, treatment and control among older people in Latin America, India and China: a 10/66 cross-sectional population-based survey. J Hypertens. 2012 Jan;30(1):177-87.

18. Lerner AG, Bernabe-Ortiz A, Gilman RH, Smeeth L, Miranda JJ. The 'rule of halves' does not apply in Peru: Awareness, treatment, and control of hypertension and diabetes in rural, urban and rural-to-urban migrants. Crit Pathw Cardiol. 2013 Jun;12(2):53-8.

19. Aquado- Fabián E, Arias-Guisado $M$ Sarmiento-Almidón G, Danjoy-Leon D. Asociación entre conocimiento y autocuidado sobre hipertensión arterial en pacientes hipertensos, consultorio de cardiología,Hospital Nacional. Rev enferm Herediana. 2014;7(2):132-39.

20. Zavala-Loayza JA, Benziger CP, Cárdenas MK, Carrillo-Larco RM, Bernabé-Ortiz 
A, et al. Characteristics Associated With Antihypertensive Treatment and Blood Pressure Control: A Population-Based Follow-Up Study in Peru. Glob Heart. 2016 Mar;11(1):109-19.

21. Rivas Chávez J, Gutiérrez Villafuerte C, Rivas Legua J. Tratamiento y costos farmacologicos de la hipertension arterial no complicada en un hospital regional. Revista Peruana de Cardiología.2008; 34(3): 181-90.

22. Silva $H$, Hernandez-Hernandez $R$, Vinueza R, Velasco M, Boissonnet CP, et al. Cardiovascular Risk Awareness, Treatment, and Control in Urban Latin America. Am J Ther. 2010 MarApr;17(2):159-66.

23. Bravo J, Sánchez J, Sosa H, Díaz-Herrera J, Miyahira J. Características clínicas de las gestantes con hipertensión arterial crónica atendidas en un hospital general de Lima. Rev Med Hered. 2013; 24:287-92.

24. Carhuallanqui R, Diestra-Cabrera G, Tang-Herrera J, Málaga G. Adherencia al tratamiento farmacológico en pacientes hipertensos atendidos en un hospital general. Rev Med Hered 2010;21:197-201.

25. Fernández-Arias $M$, Acuna-Villaorduna, Miranda JJ, Diez-Canseco F, Malaga G. Adherence to Pharmacotherapy and Medication-Related Beliefs in Patients with Hypertension in Lima, Peru. PLoS One. 2014 Dec 3;9(12):e112875.

26. Rivas Torres G, Pino Delgadoy MA, Osada Liy JE. Programas de hipertensión arterial: gran asistencia y baja adherencia. Rev Colomb Cardiol. 2016;23(6):578-79.

27. Lloyd-Sherlock P, Beard J, Minicuci N, Ebrahim S, Chatterji S. Hypertension among older adults in low- and middleincome countries: prevalence, awareness and control. Int J Epidemiol. 2014 Feb;43(1):116-28.

28. Camacho PA, Gomez-Arbelaez D, Molina DI, Sanchez G, Arcos E, et al. Social disparities explain differences in hypertension prevalence, detection and control in Colombia. J Hypertens. 2016 Dec;34(12):2344-52.

29. Ene-Iordache B, Perico N, Bikbov B, Carminati S, Remuzzi A, et al. Chronic kidney disease and cardiovascular risk in six regions of the world (ISN-KDDC): a cross-sectional study. Lancet Glob Health. 2016 May;4(5):e307-19.

30. Instituto Nacional de estadística e Informática. [Internet]. Lima. PERÚ: Enfermedades no transmisibles $y$ transmisibles, 2016. [Citado el 18 de Julio del 2017]. Disponible en: http://www. inei.gob.pe/media/MenuRecursivo/ publicaciones_digitales/Est/Lib1432/ index.html.

31. Sorlie PD, Allison MA, Avilés-Santa ML, Cai J, Daviglus ML, et al. Prevalence of hypertension, awareness, treatment, and control in the Hispanic Community Health Study/Study of Latinos. Am J Hypertens. 2014 Jun;27(6):793-800.

Correspondencia: Percy Herrera Añazco

Dirección: Olavegoya 1879 dpto 701 Jesus Maria. Lima, Perú.

Correo electrónico:silamud@gamail.com 